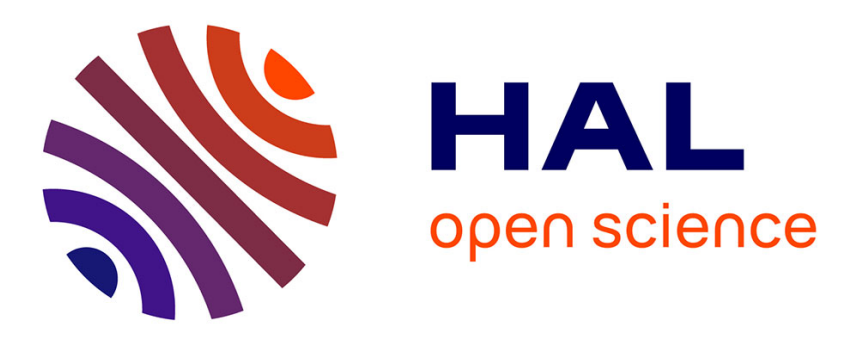

\title{
Canonical polyadic decomposition of hyperspectral patch tensors
}

Miguel Angel Veganzones, Jérémy E Cohen, Rodrigo Cabral Farias, Konstantin Usevich, Lucas Drumetz, Jocelyn Chanussot, Pierre Comon

\section{To cite this version:}

Miguel Angel Veganzones, Jérémy E Cohen, Rodrigo Cabral Farias, Konstantin Usevich, Lucas Drumetz, et al.. Canonical polyadic decomposition of hyperspectral patch tensors. EUSIPCO 2016 - 24th European Signal Processing Conference, Aug 2016, Budapest, Hungary. pp.2176-2180, 10.1109/EUSIPCO.2016.7760634 . hal-01382362

\section{HAL Id: hal-01382362 \\ https://hal.science/hal-01382362}

Submitted on 17 Oct 2016

HAL is a multi-disciplinary open access archive for the deposit and dissemination of scientific research documents, whether they are published or not. The documents may come from teaching and research institutions in France or abroad, or from public or private research centers.
L'archive ouverte pluridisciplinaire HAL, est destinée au dépôt et à la diffusion de documents scientifiques de niveau recherche, publiés ou non, émanant des établissements d'enseignement et de recherche français ou étrangers, des laboratoires publics ou privés. 


\title{
CANONICAL POLYADIC DECOMPOSITION OF HYPERSPECTRAL PATCH TENSORS
}

\author{
M.A. Veganzones ${ }^{a}$, J.E. Cohen ${ }^{a}$, R. Cabral Farias ${ }^{a}$, K. Usevich ${ }^{a}$, L. Drumetz ${ }^{b}$, J. Chanussot $^{a, c}$, P. Comon $^{a}$ \\ ${ }^{\mathrm{a}}$ CNRS, ${ }^{\mathrm{b}}$ UJF, Gipsa-Lab, Univ. Grenoble Alpes, F-38000 Grenoble, France \\ ${ }^{c}$ Faculty of Electrical and Computer Engineering, University of Iceland, Iceland
}

\begin{abstract}
Spectral unmixing (SU) is one of the most important and studied topics in hyperspectral image analysis. By means of spectral unmixing it is possible to decompose a hyperspectral image in its spectral components, the so-called endmembers, and their respective fractional spatial distributions, so-called abundance maps. The Canonical Polyadic (CP) tensor decomposition has proved to be a powerful tool to decompose a tensor data onto a few rank-one terms in a multilinear fashion. Here, we establish the connection between the $\mathrm{CP}$ decomposition and the SU problem when the tensor data is built by stacking small patches of the hyperspectral image. It turns out that the CP decomposition of this hyperspectral patch-tensor is equivalent to solving a blind regularized Extended Linear Mixing Model (ELMM).
\end{abstract}

Index Terms - Spectral unmixing, extended linear mixing model, Canonical Polyadic, nonnegative tensor decomposition, patch tensor.

\section{INTRODUCTION}

Imaging spectroscopy [1] (a.k.a. hyperspectral imaging) is concerned with the measurement, analysis, and interpretation of spectra acquired from a given scene or object [2]. Given a nonnegative hyperspectral image, $\boldsymbol{X} \in \mathbb{R}_{+}^{N \times L}$, where $N$ denotes the number of pixels and $L$ the number of spectral bands, spectral unmixing (SU) aims at estimating the spectral signatures of the materials present in the image and their spatial distributions, known respectively as endmembers and fractional abundances. This analysis ultimately permits a sub-pixel resolution. Usually, a linear mixing model (LMM) is considered:

$$
\boldsymbol{X}=\boldsymbol{\Phi} \boldsymbol{S}^{\top}+\boldsymbol{E},
$$

where $\boldsymbol{S} \in \mathbb{R}_{+}^{L \times R}$ denotes the matrix of endmembers, each of the $R$ columns representing the spectral signature of a given macroscopic material, $\boldsymbol{\Phi} \in \mathbb{R}_{+}^{N \times R}$ denotes the matrix of fractional abundances corresponding to each of the $R$ endmembers; and $\boldsymbol{E} \in \mathbb{R}^{N \times L}$ denotes additive noise. The endmembers are usually extracted from the data using one of the many endmember extraction algorithms available in the

This work has received funding from the European Research Council under the European Community's Seventh Framework Programme FP7/20072013 Grant Agreement no. 320594, "DECODA". literature [3]. Then, the abundances are estimated by solving an inverse problem. For instance, the full constrained least squares unmixing (FCLSU) is defined as:

$$
\begin{gathered}
\operatorname{argmin}\left\|\boldsymbol{X}-\boldsymbol{\Phi} \boldsymbol{S}^{\top}\right\|_{F}^{2} \\
\text { w.r.t. } \boldsymbol{\Phi} \\
\text { s.t. } \boldsymbol{\Phi} \succeq 0, \boldsymbol{\Phi} \mathbf{1}_{R}=\mathbf{1}_{N},
\end{gathered}
$$

with $\mathbf{1}_{K}$ is an unitary vector of size $K$.

Sometimes, the LMM is insufficient, especially when the macroscopic materials features spectral variabilities due to illumination inhomogeneities, topography and other effects. Recently, the extended LMM (ELMM) [4] was proposed to address the spectral variability issue:

$$
\boldsymbol{X}=\boldsymbol{\Phi} f_{k}(\boldsymbol{S})^{\top}+\boldsymbol{E}
$$

The function $f(\cdot)$ is a mapping $f: \mathbb{R}_{+}^{L \times R} \rightarrow \mathbb{R}_{+}^{L \times R}$ that accounts for the spectral variability of the endmembers. The $k$ index of the spectral variability function accounts for different flavours of the ELMM. For instance, the spectral variability function could vary across the pixels.

We propose to use tensor analysis [5] (a.k.a. multiway or multiarray analysis) to decompose the hyperspectral image in a few rank-one terms, by means of the Canonical Polyadic (CP) model [6], sometimes coined Candecomp/Parafac [7]. Recently, we have given evidence that the CP decomposition of hyperspectral time series and multiangle acquisitions could be understood as the multilinear spectral unmixing of the data [8]. However, it is not possible to apply this analysis directly to a single hyperspectral image. Some authors have proposed to consider the hyperspectral data cube as a tensor representation of the data, where the two spatial dimensions, i.e., rows and columns of the image, define two different ways of the tensor $[9,10]$. However, this representation is of little use for the CP decomposition since the resulting tensor is not low rank. Thus, we propose to build what we have termed a patch-tensor representation of a hyperspectral image by stacking small patches of the image. The resulting tensor, $\mathcal{X} \in \mathbb{R}_{+}^{N \times L \times B}$, has a third way containing the $B$ neighbouring pixels of each of the $N$ pixels of the image. In Sec. 2, we provide a detailed explanation of the proposed patch-tensor representation.

The nonnegative $\mathrm{CP}$ decomposition allows to decompose the patch-tensor, $\mathcal{X}$, into a multilinear combination of $R$ non- 
negative factor matrices $\boldsymbol{A}, \boldsymbol{B}$ and $\boldsymbol{C}$, of size $N \times R, L \times R$ and $B \times R$, respectively, and :

$$
\mathcal{X}_{i j k}=\sum_{r=1}^{R} A_{i r} B_{j r} C_{k r},
$$

where the scaling of factors has been pulled in $C$. The rank of tensor $\mathcal{X}$ is defined as the minimal number $R$ of terms necessary for the equality above to hold exactly [5]. A shorter expression can be employed to denote decomposition (4) using a diagonal tensor of ones, denoted $\mathcal{I}$, of size $R \times R \times R$, i.e. $\mathcal{X}=(\boldsymbol{A} \otimes \boldsymbol{B} \otimes \boldsymbol{C}) \mathcal{I}$, where $\otimes$ is the tensor product.

In practice, the data tensor is subject to modeling errors or measurement noise, and it is convenient to find its best rank- $R$ approximation by minimizing the following objective function

$$
\Upsilon(\boldsymbol{A}, \boldsymbol{B}, \boldsymbol{C})=\|\mathcal{X}-(\boldsymbol{A} \otimes \boldsymbol{B} \otimes \boldsymbol{C}) \mathcal{I}\|,
$$

for some well chosen norm, instead of attempting to compute the exact CP decomposition of $\mathcal{X}$ with, unavoidably, a large rank. It is now known that tensors of order 3 or larger do not always admit a best rank- $R$ approximate when $R>1$, especially in $\mathbb{R}$ or $\mathbb{C}$. But fortunately, it has been shown in [11] that this obstacle does no longer hold for nonnegative tensors, and that the problem is well-posed in $\mathbb{R}^{+}$: best lower rank nonnegative approximates always exist and are generically unique under mild conditions on $R$ [12].

We show in Sec. 3, that the nonnegative CP decomposition of a hyperspectral patch-tensor is equivalent to solving a blind regularized version of the ELMM model (3), where rank-one factor estimates correspond to spatial abundances, endmembers and local spectral variabilities. The CP decomposition is a blind technique in the sense that no a priori information is needed, i.e., the spectral signatures of the materials in the image are assumed unknown. We further discuss on some preliminary experimental results using synthetic data in Sec. 4, and we finally give some conclusions in Sec. 5.

\section{PATCH-TENSOR REPRESENTATION}

Let us denote by $\boldsymbol{x}_{i} \in \mathbb{R}_{+}^{L}$, the $i$-th pixel, $1 \leq i \leq N$, of a hyperspectral image. For each pixel $\boldsymbol{x}_{i}$, a patch is defined by the matrix $\boldsymbol{P}_{i}=\left[\boldsymbol{x}_{i_{1}}, \boldsymbol{x}_{i_{2}}, \ldots, \boldsymbol{x}_{i_{B}}\right]$, of $B$ pixels $\boldsymbol{x}_{i_{k}}$ in the neighbourhood of $\boldsymbol{x}_{i}$, including $\boldsymbol{x}_{i}=\boldsymbol{x}_{i_{1}}$. A neighbourhood of $\boldsymbol{x}_{i}$ can be the set of pixels adjacent to $\boldsymbol{x}_{i}$ using a sliding window (see Fig. 1). Each matrix $\boldsymbol{P}_{i} \in \mathbb{R}_{+}^{L \times B}$ is stacked to define a slice of the patch-tensor, $\mathcal{X} \in \mathbb{R}_{+}^{N \times L \times B}$. The above formulation of the patchtensor is equivalent to stacking $B$ images $\boldsymbol{X}^{(k)} \in \mathbb{R}_{+}^{N \times L}$, $1 \leq k \leq B$, obtained by shifting the original hyperspectral image along the neighbourhood, i.e. a shifted image is defined by $\boldsymbol{X}^{(k)}=\left[\boldsymbol{x}_{\left(1+d_{k}\right)}, \ldots, \boldsymbol{x}_{\left(N+d_{k}\right)}\right]^{\top}$, where $d_{k}$ denotes a spatial displacement from the center of the patch to the position of the $k$-th element in the patch. This yields zero-padding along the border of the hyperspectral image.

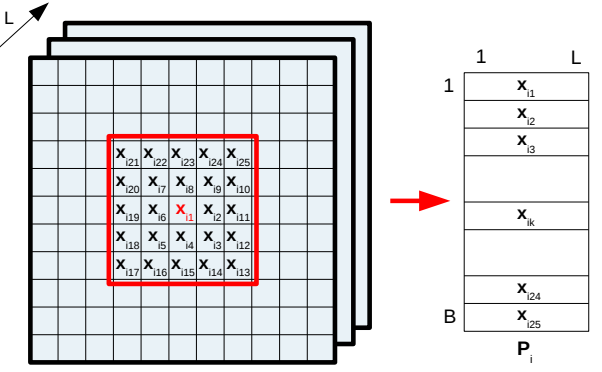

Fig. 1. Example of a $5 \times 5$ patch using a sliding window centred in $\mathbf{x}_{i}$, and the corresponsing matrix patch, $\boldsymbol{P}_{i}$.

\section{NONNEGATIVE CP DECOMPOSITION OF THE PATCH-TENSOR}

\subsection{Compressed nonnegative $\mathrm{CP}$ decomposition}

Given the patch-tensor, $\mathcal{X} \in \mathbb{R}_{+}^{N \times L \times B}$, its approximation by a nonnegative $\mathrm{CP}$ decomposition (4) is formulated as:

$$
\begin{gathered}
\operatorname{argmin}\|\mathcal{X}-(\boldsymbol{A} \otimes \boldsymbol{B} \otimes \boldsymbol{C}) \mathcal{I}\|_{F}^{2} \\
\text { w.r.t. } \boldsymbol{A}, \boldsymbol{B}, \boldsymbol{C} \\
\text { s.t. } \boldsymbol{A} \succeq 0, \boldsymbol{B} \succeq 0, \boldsymbol{C} \succeq 0,
\end{gathered}
$$

where $\|\cdot\|_{F}$ denotes the Frobenius norm. This problem is highly non-convex, yet many algorithms provide rather precise but costly computation.

An approach to handle large tensor decomposition is through the use of compression. The general idea is that the original patch-tensor $\mathcal{X}$ can be equivalently represented by a compressed version of it, $\mathcal{X}_{c}$, with reduced dimensions $N_{c} \times D_{c} \times T_{c}$. The compressed tensor is then decomposed by solving

$$
\begin{aligned}
\operatorname{argmin} \Upsilon= & \left\|\boldsymbol{\mathcal { X }}_{c}-\left(\boldsymbol{A}_{c} \otimes \boldsymbol{B}_{c} \otimes \boldsymbol{C}_{c}\right) \mathcal{I}\right\|_{F}^{2} \\
& \text { w.r.t. } \boldsymbol{A}_{c}, \boldsymbol{B}_{c}, \boldsymbol{C}_{c},
\end{aligned}
$$

where $\boldsymbol{A}_{c}, \boldsymbol{B}_{c}, \boldsymbol{C}_{c}$ are compressed versions of the original factor matrices. Note that, after the compressed factors are obtained, a decompression operation is carried out to recover the factors in the original dimensions.

Authors in [13] provide two algorithms, a compressed conjugate gradient (CCG) and a Projected and Compressed Alternate Least Squares (ProCo-ALS) to solve (7) when the compression is done using an approximate High Order Singular Value Decomposition (HOSVD):

$$
\boldsymbol{\mathcal { X }}_{i j k} \approx \sum_{l m n}^{N_{c}, D_{c}, T_{c}} \boldsymbol{U}_{i l} \boldsymbol{V}_{j m} \boldsymbol{W}_{k n}\left[\boldsymbol{\mathcal { X }}_{c}\right]_{i j k},
$$

or using the same notation as in the $\mathrm{CP}$ model

$$
\mathcal{X} \approx(\boldsymbol{U} \otimes \boldsymbol{V} \otimes \boldsymbol{W}) \boldsymbol{\mathcal { X }}_{c}
$$

where $\boldsymbol{U}, \boldsymbol{V}$ and $\boldsymbol{W}$ are matrices with orthogonal unit-norm columns. These algorithms solve the optimization problem 
defined in (7) enforcing the nonnegativity of the factors in the unconstrained domain:

$$
\boldsymbol{A} \approx \boldsymbol{U} \boldsymbol{A}_{c} \succeq 0, \quad \boldsymbol{B} \approx \boldsymbol{V} \boldsymbol{B}_{c} \succeq 0, \quad \boldsymbol{C} \approx \boldsymbol{W} \boldsymbol{C}_{c} \succeq 0 .
$$

Here, we make use of a modified version of ProCo-ALS to include the abundances sum-to-one physical constraint usually employed in spectral unmixing:

$$
\boldsymbol{A} \mathbf{1}_{R}=\mathbf{1}_{N}
$$

as in (2). The modified ProCo-ALS algorithm works by projecting onto the unitary simplex instead of projecting onto the nonnegative orthant.

\subsection{Regularized extended linear mixing model (rELMM) equivalence}

Here, we show that the $\mathrm{CP}$ decomposition (6) of the patchtensor, $\mathcal{X}$, is equivalent to a regularized version of an ELMM formulation (3). Given the tensor slice, $\mathcal{X}_{:,, k} \equiv \boldsymbol{X}^{(k)}$, where the colon symbol denotes all the elements of the corresponding way, its $\mathrm{CP}$ decomposition is given by:

$$
\mathcal{X}_{:,:, k} \approx \sum_{r=1}^{R} \boldsymbol{A}_{:, r} \boldsymbol{B}_{:, r}^{\top} \boldsymbol{C}_{k, r}=\boldsymbol{A} \boldsymbol{\Lambda}_{k} \boldsymbol{B}^{\top}
$$

$\boldsymbol{\Lambda}_{k}$ being a $R \times R$ diagonal matrix with entries $\operatorname{diag}\left(\boldsymbol{\Lambda}_{k}\right)=$ $\boldsymbol{C}_{k,:}$. If we consider that factor matrices $\boldsymbol{A}$ and $\boldsymbol{B}$ are respectively estimates of the abundance matrix $\boldsymbol{\Phi}$ and the set of endmembers $\boldsymbol{S}$, then the CP model in (12) is equivalent to:

$$
\boldsymbol{X}^{(k)} \approx \hat{\boldsymbol{\Phi}} \boldsymbol{\Lambda}_{k} \hat{\boldsymbol{S}}^{\top}
$$

Thus, the nonnegative CP decomposition of the patchtensor $\mathcal{X}$ can be reformulated as a blind joint solution to the following $B$ ELMM models:

$$
\boldsymbol{X}^{(k)} \approx \boldsymbol{\Phi} f_{k}^{\top}(\boldsymbol{S}), k=1, \ldots, B,
$$

where the spectral variability function varies for each of the $B$ slices:

$$
f_{k}(\boldsymbol{S})=\boldsymbol{S} \boldsymbol{\Lambda}_{k}, k=1, \ldots, B .
$$

That is, the $\mathrm{CP}$ decomposition provides a blind solution to the following optimization problem:

$$
\begin{gathered}
\operatorname{argmin}\left\|\boldsymbol{X}^{(1)}-\boldsymbol{\Phi} f_{1}^{\top}(\boldsymbol{S})\right\|_{F}^{2}+\ldots+\left\|\boldsymbol{X}^{(B)}-\boldsymbol{\Phi} f_{B}^{\top}(\boldsymbol{S})\right\|_{F}^{2} \\
\text { w.r.t. } \boldsymbol{\Phi}, \boldsymbol{S}, \boldsymbol{\Lambda}_{1}, \ldots, \boldsymbol{\Lambda}_{B} \\
\text { s.t. } \boldsymbol{\Phi} \succeq 0, \boldsymbol{\Phi} \mathbf{1}_{R}=\mathbf{1}_{N}, \boldsymbol{S} \succeq 0, \boldsymbol{\Lambda}_{1} \succeq \mathbf{0}, \ldots, \boldsymbol{\Lambda}_{B} \succeq \mathbf{0} .
\end{gathered}
$$

The model in (16) corresponds to a regularized ELMM model with the spectral variability given by a diagonal matrix of scaling factors. The regularization imposes that, for each pixel in the image, the pixels in its neighbourhood should have the same materials and in the same proportions, up to a modulation of their spectra given by different local scaling factors. This suggests setting $B$ to a small value, since the regularization should be valid only for small neighbourhoods. In practice, these constraints impose smoothness on the spatial factors. Therefore, the scaling factors defined by the factor matrix $\boldsymbol{C}$ could be understood as local patterns of spectral variabilities affecting each material in the image.

\section{EXPERIMENTAL VALIDATION}

\subsection{Synthetic images}

We chose two sets, of three reference endmembers each, corresponding to signatures of minerals from the United States Geological Survey (USGS) spectral library, comprising 224 spectral bands in the visible and near-IR. The first dataset, $S_{1}$, presents no correlated endmembers, while the second dataset, $S_{2}$, has two endmembers that are highly correlated. For each dataset, we simulated 10 synthetic hyperspectral images as follows. We generated $200 \times 200$ abundance maps using Gaussian Fields complying with the abundances sum-to-one constraint. Note that these abundance maps comprise only one pure pixel for each material, and around $5 \%$ of the pixels have an abundance coefficient superior to 0.9 for any material. We also generated spectral variability maps for each endmember using mixtures of Gaussians. Then, the pixel-dependent endmember instances were generated by multiplying the references by the corresponding spectral variability scaling factors (the achievable values are chosen so that no reflectance value becomes higher than 1 , and so that the scaling factors range from 0.75 to 1.25$)$. Then for each pixel, the mixture was performed using the linear mixing model (1), and finally we added a $30 \mathrm{~dB}$ white Gaussian noise to the image. The patch-tensor is built using a $5 \times 5$ sliding window, that is, $B=25$.

\subsection{Methodology and results}

For each dataset and synthetic image, we run 20 Monte Carlo runs of the ProCo-ALS algorithm on the patch tensor, ensuring random initial factors hold the nonnegativity and, for the spatial factor, the abundances sum-to-one constraint. For the sake of comparison, we also run 20 Monte Carlo runs of the Vertex Component Analysis [14] endmember extraction algorithm to estimate the spectral signatures from the original image, keeping the ones with highest volume. Then, we solved the FCLSU (2) to estimate the abundances. We compared the results to the actual endmembers and abundances, using the angular distance and the average root mean squared error, respectively. In Fig. 2, we compared the worst case ProCoALS and VCA/FCLSU results. The VCA is not very affected by the spectral variability, and since there are pure pixels in the image, the estimated endmembers are very close to the actual ones. However, the abundance estimates obtained by FCLSU could be seriously affected yielding to poor results. The ProCo-ALS obtains better estimates of the abundances, with a small loss in accuracy in the endmembers estimation. 
The effect of the endmembers correlation is clear when looking at the results for the two datasets, $S_{1}$ and $S_{2}$. On one hand, the presence of correlated endmembers yields reduced dependency on the initialization conditions. On the other hand, abundance estimates are better when data have no correlated endmembers.

Fig. 3 shows a comparison of the best results obtained by the ProCo-ALS among the 20 random initializations to the VCA/FCLSU, in terms of either endmembers or abundances estimation accuracy for the uncorrelated dataset, $S_{1}$, and the correlated one, $S_{2}$. For the former, the estimation of the abundances is always good, and the estimation of the endmembers is good enough (less than 5 degrees). For the latter, the accuracy of abundances depends on the accuracy of endmember estimation, and in some cases, the high correlation of the actual endmembers makes their accurate estimation a too difficult problem for a completely blind approach.

\subsection{Discussion}

Preliminary results show the potential of the proposed CP decomposition of hyperspectral patch-tensors for solving a blind spectral unmixing, in the presence of spectral variability. Still, some issues should be further addressed. The presence of correlated endmembers reduces the capacity of the $\mathrm{CP}$ decomposition to correctly identify the materials and estimate their fractional abundances. Also, it is not clear how to choose the best solution among many random initializations in practical situations, since the tensor reconstruction error appears to be uncorrelated to endmember and abundance estimation accuracy. Moreover, we are working on a modification of the proposed method to relax the regularization terms in (16), and to propose a semi-blind $\mathrm{CP}$ decomposition, where the spectral factors are regularized to improve their identifiability.

\section{CONCLUSIONS}

The present paper introduces two contributions. On one hand, we have proposed a patch-tensor representation of hyperspectral images, by stacking patches of the image in the third way of the tensor. On the other hand, We showed the equivalence between the $\mathrm{CP}$ decomposition of the proposed hyperspectral patch-tensor to a regularized version of the extended linear mixing model (ELMM) for spectral unmixing. Thus, the proposed approach aims at solving a blind spectral unmixing problem in the presence of spectral variability. We provided some preliminary results using synthetic data, and highlighted some issues and possible solutions. Further work will focus on relaxing the regularization terms of the $\mathrm{CP}$ decomposition equivalent ELMM, a semi-blind approach, and on providing a criterion to choose a good initialization. Also, we are working on giving evidence using realistic synthetic images and real datasets.

\section{REFERENCES}

[1] A. F. H. Goetz, G. Vane, J. E. Solomon, and B. N. Rock, "Imaging spectrometry for earth remote sensing," Science, vol. 228, no. 4704, pp. 1147-1153, 1985.

[2] A. Plaza, J. A. Benediktsson, J. W. Boardman, J. Brazile, L. Bruzzone, G. Camps-Valls, J. Chanussot, M. Fauvel, P. Gamba, A. Gualtieri, M. Marconcini, J. C. Tilton, and G. Trianni, "Recent advances in techniques for hyperspectral image processing," Rem. Sens. of Env., vol. 113, Supplement 1, pp. 110-122, 2009.

[3] J. M. Bioucas-Dias, A. Plaza, N. Dobigeon, M. Parente, D. Qian, P. Gader, and J. Chanussot, "Hyperspectral unmixing overview: Geometrical, statistical, and sparse regressionbased approaches," IEEE J. Sel. Topics Appl. Earth Observ., vol. 5, no. 2, pp. 354-379, Apr. 2012.

[4] M.A. Veganzones, L. Drumetz, G. Tochon, M. Dalla Mura, A. Plaza, J. Bioucas, and J. Chanussot, "A new extended linear mixing model to address spectral variability," in 6th IEEEWHISPERS, 2014.

[5] P. Comon, "Tensors : A brief introduction," IEEE Sig. Proc. Mag., vol. 31, no. 3, pp. 44-53, May 2014.

[6] F. L. Hitchcock, "The expression of a tensor or a polyadic as a sum of products," J. Math. Physics, vol. 6, no. 1, pp. 165-189, 1927.

[7] H. A. L. Kiers, "Towards a standardized notation and terminology in multiway analysis," J. Chemometrics, pp. 105-122, 2000.

[8] M.A. Veganzones, J.E. Cohen, R. Cabral Farias, J. Chanussot, and P. Comon, "Nonnegative tensor cp decomposition of hyperspectral data," IEEE Trans. Geosci. Remote Sens., 2015, to appear.

[9] L. Zhang, L. Zhang, D. Tao, and X. Huang, "Tensor discriminative locality alignment for hyperspectral image spectralspatial feature extraction," IEEE Trans. Geo. Rem. Sens., vol. 51, no. 1, pp. 242-256, 2013.

[10] S. Bourennane, C. Fossati, and A. Cailly, "Improvement of classification for hyperspectral images based on tensor modeling," IEEE Geosci. Remote Sens. Lett., vol. 7, no. 4, pp. 801-805, 2010.

[11] L.-H. Lim and P. Comon, "Nonnegative approximations of nonnegative tensors," J. Chemometrics, vol. 23, no. 7-8, pp. 432-441, 2009.

[12] Y. Qi, P. Comon, and L.-H. Lim, "Uniqueness of nonnegative tensor approximations," IEEE Trans. Inf. Theory, 2016, to appear, arxiv:1410.8129.

[13] J.E. Cohen, R.C. Farias, and P. Comon, "Fast decomposition of large nonnegative tensors," Signal Processing Letters, IEEE, vol. 22, no. 7, pp. 862-866, July 2015.

[14] J.M.P. Nascimento and J.M. Bioucas Dias, "Vertex component analysis: a fast algorithm to unmix hyperspectral data," IEEE Trans. Geosci. Remote Sens., vol. 43, no. 4, pp. 898-910, April 2005. 


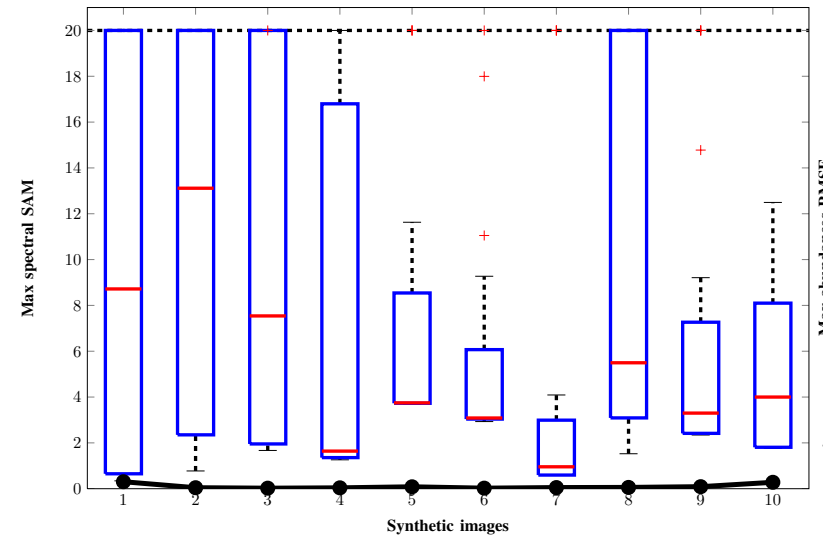

(a)

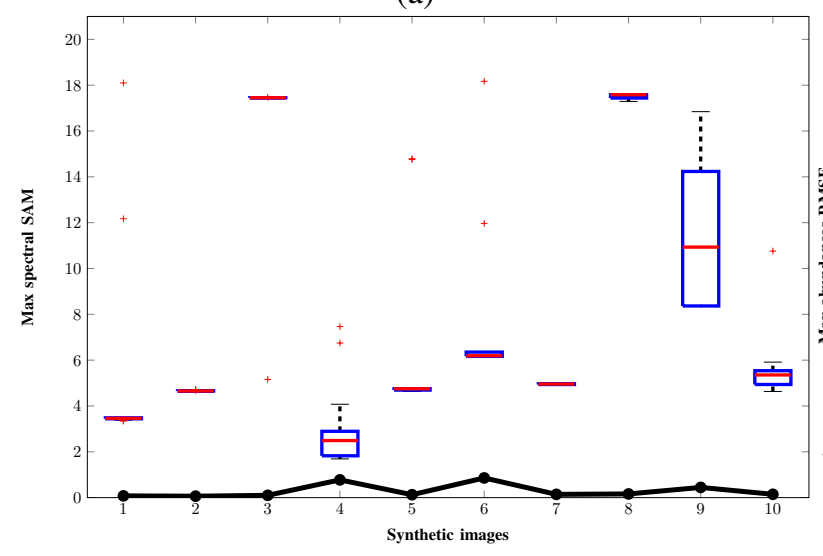

(c)

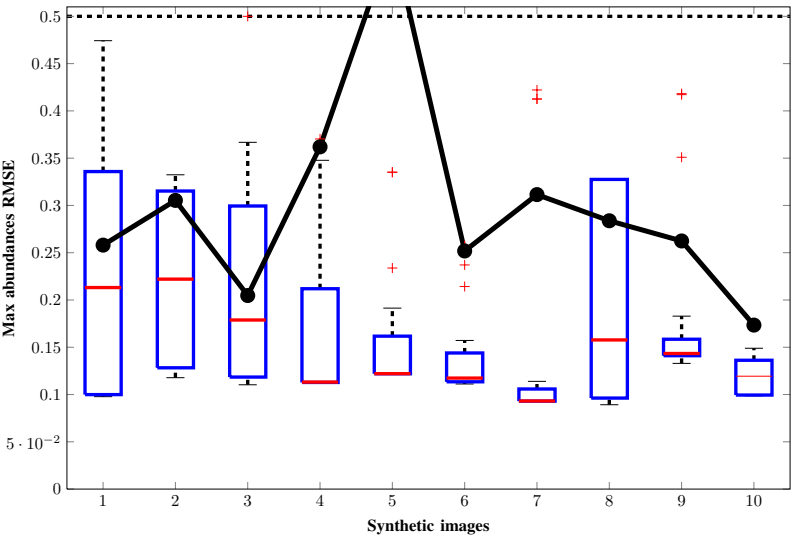

(b)

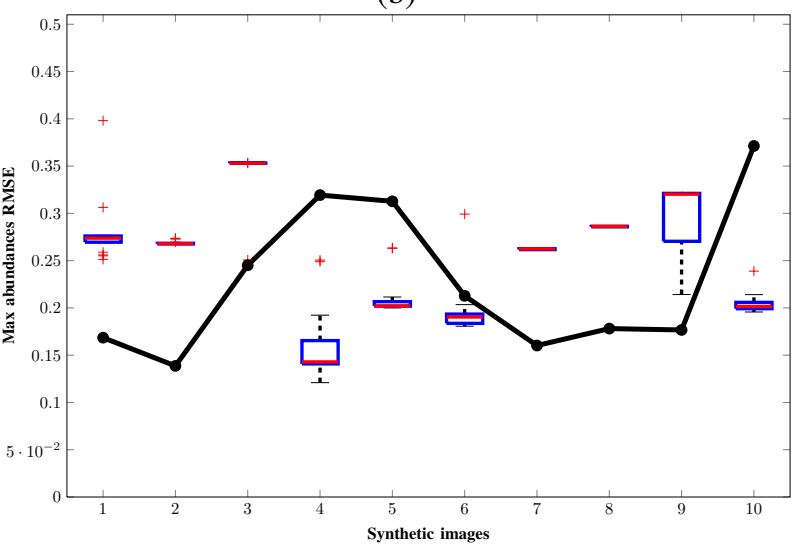

(d)

Fig. 2. . Box plot of the 20 runs worst case results of the CP decomposition using ProCo-ALS and random initializations, compared to the results obtained by VCA/FCLSU (in black): (a) Spectral angular distance for $S_{1}$, (b) Abundances average RMSE for $S_{1}$, (c) Spectral angular distance for $S_{2}$, and (b) Abundances average RMSE for $S_{2}$.

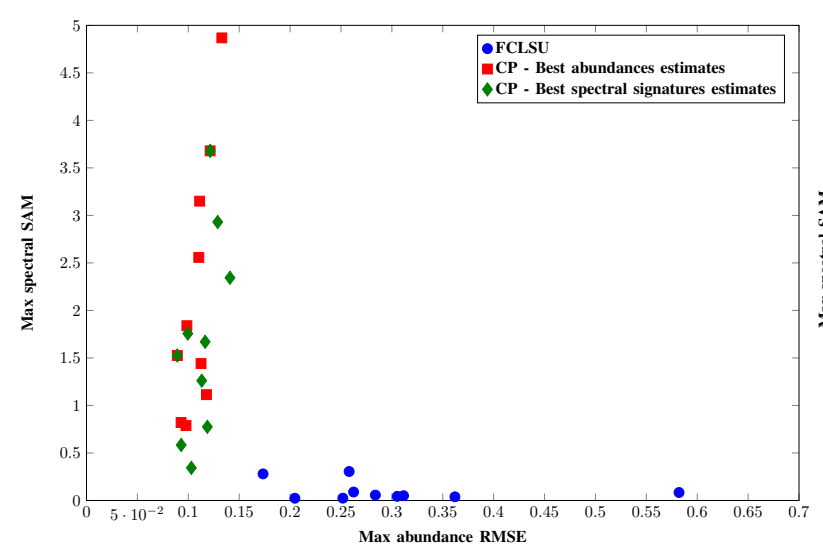

(a)

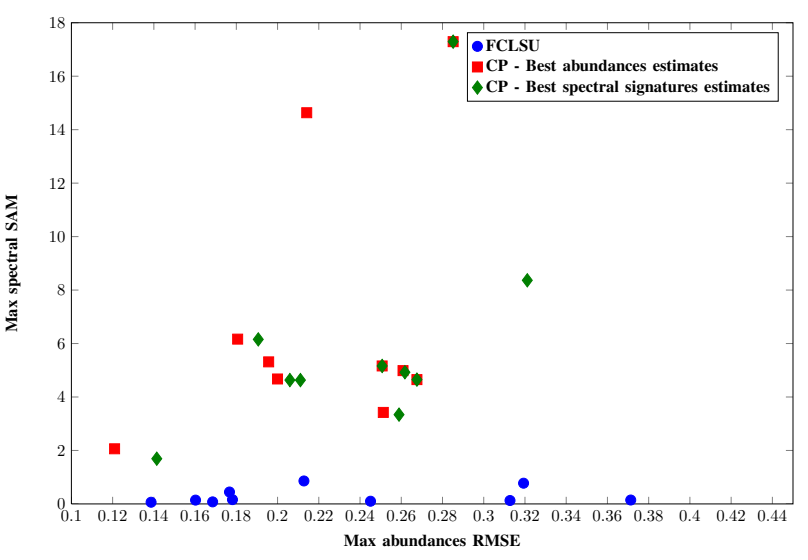

(b)

Fig. 3. Abundances accuracy versus endmembers accuracy for the best $\mathrm{CP}$ results among the 20 random initializations, using ProCo-ALS, in terms of abundances accuracy (red squares) and endmembers accuracy (green diamonds), compared to the VCA/FCLSU (blue dots): (a) Uncorrelated dataset $S_{1}$, (b) Correlated dataset $S_{2}$. 